of the bones could be seen and felt. The soft parts about the elbow were much bruised and lacerated. There was also a fracture of the radius just above the wrist. As Dr. Ingalls was absent, Dr. Homans saw the patient, and advised immediate amputation or resection, to which the patient would not consent. Dr. Homans then reduced the dislocation, the patient being etherized. The wound was packed with charpie soaked in carbolic oil, and a rectangular external splint applied. The second day the elbow was much swollen and the wound offensive. Dr. Ingalls advised amputation or excision, but the patient declined any operative interference with the joint. Contrary to expectation, from this time the condition of the arm steadily improved, the only complication being the formation of an abscess on the inner side of the fore-arm, just below the elbow, which discharged through a spontaneous opening into the original wound. There was considerable discharge for about three weeks. On the forty-third day the opening had bealed, and the arm was put in a stiff bandage, the elbow being flexed at a right angle. The Colles's fracture received no treatment but rest. The patient was discharged on the forty-eighth day, with the bandage still on. 'Two weeks after her discharge she had the stiff bandage removed in the out-patient department. The fore-arm could not be flexed or extended, but the hand could to a slight degree be supinated and pronated. There was some inward projection of the lower end of the ulna. There was considerable motion at the wrist. When last seen she could make but little use of her hand or arm.

October 4th, eighty-eight days after the accident, the arm was examined, and arm and hand were found in about the same condition as above indicated. The patient declared that she suffered much pain throughout the limb, and that little bits of bone had come away. If this be true, they must have come through the yet tender cicatrix which soon closed after their passage.

\title{
POISONING BY CORROSIVE SUBLIMATE AND BY CARBOLIC ACID.
}

Mr. Editor, - Two cases of poisoning have come under my immediate notice. In one I was permitted to make a post-mortem examination, which may render a report useful. The other case, as it was from carbolic acid, about which as a poison little seems to have been said, may serve to impress upon your readers a very important property of this drug.

Both the cases occurred at the St. Louis Female Hospital, under the care of Dr. P. V. Schenck, resident physician, and myself, assistant physician. One of the patients, Alice W., who was convalescing from intermittent fever, complained of constipation, and a dose of sulphate of magnesium was ordered. The nurse had solutions of sulphate of magnesium and of corrosive sublimate in similar bottles, but plainly labeled; by mistake, however, she gave the mercuric salt in a dose of about sixty grains.

I saw the patient probably within twenty or twenty-five minutes after the poison was taken. She had vomited freely, but large quantities of water and milk were given, and emesis produced, which was further encouraged by sulphate of zinc and ipecac. About an hour and a half after the accident, Dr. 
Schenck, who had been absent upon business, returned, and two ounces of the white of eggs were given every ten minutes, till about twelve ounces had been taken.

Some three or four hours after the poison was administered there was an abundant discharge of urine and several evacuations of her bowels. The patient suffered little pain, and the following day her recovery seemed almost certain. The third day she was very weak, vomiting set in, and she seemed sinking. Bismuth in thirty-grain doses was given by the mouth; enemata of milk and whisky, two ounces each, were given every two hours, and hypodermic injections of tincture of digitalis when the heart's action grew weak.

The symptoms which characterized the case were suppression of urine, insomnia, and loss of appetite; only after several days was any abdominal tenderness developed.

Dr. Boisliniere was called in, and the acetate and chlorate of potassium were added to our treatment. Warm fomentations were applied over the hips and abdomen, and also leeches over the kidneys. I was told a small flow of urine followed the use of the leeches. Tincture of digitalis stupes were also used. On the morning of the sixth day two drachms of the fluid extract of jaborandi were ordered every hour till the patient perspired. About six P. M. she began to perspire, and the jaborandi was stopped; two hours later she died in convulsions, probably uræmic.

The post mortem was made twenty-seven hours after death. There were no signs of decomposition. There was marked hypostatic congestion of the lungs. The heart was relaxed and flabby. The osophagus and cardiac extremity of the stomach were very much congested, but there were no signs of ulceration. The alimentary tract was not opened farther, but appeared to be congested, especially the small intestines. I had no means of weighing the kidneys, but they were from one third to one half larger and heavier than normal. They had not their usual flattened appearance, but were so distended with blood as to be nearly cylindrical, and upon section the blood could be squeezed from them in large quantities. The bladder was empty.

The post mortem pointed plainly to uræmia, produced by congestion of the kidneys, and that the best treatment would have been extensive local depletion. It is also possible that we erred in giving so large a quantity of albumen, as the insoluble compound formed by mercury and albumen is soluble in an excess of albumen. It might have been better to have induced vomiting every few minutes while the eggs were being administered.

Shortly after the patient died the nurse who had made the mistake went to her room, locked the door, and took about two ounces of carbolic acid. From the time she was seen alive till her door was broken open was not more than ten or at the most fifteen minutes. During this time she seems to have washed and dressed herself, and arranged herself very carefully on the bed, and then to have taken the poison. I was the first to enter the room, and immediately felt for her pulse, but was unable to find it. She gasped once or twice afterwards, but otherwise was motionless. Her death was so sudden that at first we thought she had taken prussic acid, but it proved to have been carbolic acid. The mouth and tongue were stained with it, and the bottle which had contained it was in the room empty. Unfortunately, no post mortem was 
allowed, but we suppose that she died from cardiac paralysis due to shock. Dr. H. C. Wood mentions in his treatise on Materia Medica and Therapeutics that carbolic acid has produced death almost as quickly as prussic acid.

St. Lours.

M. Hayward Post.

\section{SHORT COMMUNICATIONS.}

\section{GRITTI'S SUPRA-CONDYLOID AMPUTATION OF THE THIGH.}

IN the Medical Record for April 12, 1879, is a paper entitled as above. In that paper it is recorded that Dr. Gouley, then an assistant surgeon in the United States army, performed a similar operation on a wounded soldier, December 13,1862, the day of the battle of Fredericksburg. It was believed by the operator to have been an original operation, and also to have been the first of the kind performed in America. But in this connection it may be of interest to relate the following:-

After the battle of Antietam (September 17, 1862) it was determined to establish upon that battlefield a hospital for such severe cases as could not be conveniently removed to the general hospitals. Dr. Bernard Van der Kieft, United States volunteer surgeon, was detailed surgeon in charge of this hospital. Among the patients was a soldier with an amputation of the right leg. After some weeks it was found that the leg must be amputated at or above the knee-joint. Dr. Van der Kieft decided to amputate at the knee-joint by " $a$ new operation" (as he said), and the operatibn was certainly new to his associates and assistants. He made a circular incision from the internal tuberosity of the tibia across the leg below the patella to the head of the fibula, dissected this flap up to about the middle of the patella, cut through the ligamentum patellæ close to the lower border of the bone, and turned back the flap. He then made the posterior flap by passing the knife beneath the femur, and cutting outward and downward. He then sawed through the femur just above the condyles, and removed a section from the posterior or internal surface of the patella by the saw. Next he adjusted the sawn surface of the patella to the sawn extremity of the femur, and adjusted the flaps in the usual manner. The patient died in about sixteen hours, on account of previous exhaustion.

In a few days the writer was ordered to his regiment (Fifth New Hampshire Volunteers), and was at the battle of Fredericksburg, December 13, 1862. The journey from Antietam to Fredericksburg required two days; hence Dr. Van der Kieft's operation was performed as early as the 9th of December, 1862.

I write this in justice to the memory of Dr. Van der Kieft, who is now dead. It detracts nothing from the fame of Dr. Gouley, affords me the great satisfaction of doing honor to my friend and superior officer, and places upon record another fact in surgical history.

It thus appears that Dr. Van der Kieft performed the second or third operation of the kind ever performed, and the first in America.

Bath, N. H., November 6, 1879.

William Child, M. D., Late Surgeon Fifth Regiment N. H. Vols.

\section{NEW PREPARATIONS.}

\section{A NEW AND CHEAP WATER-PROOF TISSUE.}

Somr years ago Dr. W. W. Keen, of Philadelphia, introduced into St. Mary's Hospital of that city a sort of waxed paper, then in the market, as a substitute for the more expensive oiled silk, gutta-percha cloth, etc. In many ways the paper was unsatisfactory. It was not water-proof, it absorbed the discharges of wounds, etc., it tore easily, etc. Last winter Dr. Keen requested Seabury and Johnson, of New York, to make for him a paper coated with a combination of paraffine and rubber. They did so with such success that the new "water-proof paper" will undoubtedly replace every possible article of similar nature heretofore used. Upon receiving a sample of the paper, prepared according to his directions, Dr. Keen subjected it to serere comparative tests, the articles brought in competition being oiled silk, oiled muslin, taffeta oiled silk, paraffine paper, waxed paper, and gutta- 